\title{
O PROCESSO DE SOCIALIZAÇÃO DOS ENFERMEIROS EM UM CENTRO DE TRATAMENTO INTENSIVO*
}

\author{
THE PROCESS OF SOCIALIZATION AN INTENSIVE CARE UNIT
}

\author{
EL PROCESO DE SOCIALIZACIÓN DE LOS ENFERMEROS EN UM \\ CENTRO DE TRATAMIENTO INTENSIVO
}

\begin{abstract}
Marisa Antonini Ribeiro Bastos**
Bastos MAR. O processo de socialização dos enfermeiros em um Centro de Tratamento Intensivo. Rev Esc Enferm USP 2001; 35(3):291-9.

\section{RESUMO}

O objetivo deste trabalho foi compreender o processo de socialização dos enfermeiros integrantes da subcultura de um Centro de Tratamento Intensivo (CTI). Acreditando que o CTI é uma subcultura do hospital e que os enfermeiros intensivistas compartilham de simbolos e significados que foram desenvolvidos através das interações sociais no contexto da Terapia Intensiva, fui buscar no interacionismo simbólico e na descrição etnográfica a fundamentação teórico - metodológica para o presente estudo. Para compreender o processo de interiorização dos universos simbólicos no CTI busquei descrever os sistemas temáticos de significados subjacentes às atividades dos enfermeiros intensivistas, através da observação participante, da entrevista etnográfica e da análise documental. Os resultados mostraram que o processo de socialização é permeado de sentimentos de insegurança, ansiedade, angústia, de incompetência e de medo, As causas destes sentimentos têm um forte componente na não vivência anterior e na lacuna da formação profissional. Este processo se dá formal e informalmente, mas é sempre referido como árduo, penoso; muitas vezes solitário; algumas vezes compartilhado,
\end{abstract}

PALAVRAS - CHAVE: Cultura organizacional, Unidades de Terapia Intensiva. Enfermagem,

\section{ABSTRACT}

The objective of this study was to understand the process of socialization of the new professionals that are part of the subculture of an intensive care unit (ICU), Considering that the ICU is a subculture of the hospital, and that the professionals share symbols and meanings that were developed through social interactions in the context of Intensive Care the symbolic interacionism and the ethnographic description were used as a theoretical and methodological basis for this study. The participant observation, the ethnographic interview and the documented analysis were used to understand the, process of internalization of the symbolic universe of the ICU, The results showed that in the socialization process quality values, discipline, commitment, sense of unit, rules and organizational values were reproduced. This process is permeated with feelings of insecurity, anxiety, distress, incompetence and fear, The causes for these feelings have a strong component in the previous nonexistence and in the gap of professional career, This process occurs in a formal and informal way but is always referred as difficult, lonely and sometimes shared.

KEYWORDS: Organizational culture, Intensive Care Unit. Nursing.

\section{RESUMEN}

El trabajo tiene como objetivo compreender el proceso de socialización de los enfermeros integrantes de la subcultura de un Centro de Tratamiento Intensivo (CTI). Reconociendo que el CTI es una subcultura del hospital y que los enfermeros intensivistas comparten de los simbolos y significados, que fuerón desarrollados a través de las interaciones sociales en el contexto de la Terapia Intensiva, fué utilizado el interacionismo simbolico y en la descrición etnografica la fundamentación teorica teorico-metodologica para el estudio. Para la comprensión del proceso del interiorización de los universos simbolicos en el CTI fuerón descritos los sistemas tematicos de significados subjacentes a las actividades de los enfermeros que trabajan en el CTI, a través de la observación participante, de la entrevista etnografica y del analisis documental. Los resultados demonstran que el proceso de socialización es permeado de sentimientos de inseguridad, ansiedad, angustia, falta de competencia y de miedo. Las causas de esos sentimientos tiene realación con la ausencia de experiencia anterior de los enfermeros y con la brecha en la formación profisional, Esto ocorré formal y informalmiente, pero esta siempre referido como una experiencia ardua, my penosa, muchas vezes aislada y algumas pocas vezes compartida.

PALABRAS-CLAVE: Cultura organizacional. Cientro de Tratamiento Intensivo, Enfermería,

\footnotetext{
* $\quad$ Projeto com apoio financeiro da FAPEMIG

** Doutora. Prof ${ }^{*}$.Adjunto do Departamento de Enfermagem Básica da Escola de Enfermagem da UFMG
} 


\section{INTRODUÇÃO}

Logo que comecei a trabalhar no CTI, me despertaram a minha atenção as peculiaridades daquele setor e dos profissionais de saúde que ali atuavam, pois observava a existência de uma identidade compartilhada por seus membros, identidade esta, constituída por valores, idéias, significados, "ao lado de comportamentos e símbolos bastante visiveis" (1).

Os Centros de Tratamento Intensivo, considerados como um ambiente fechado com grande absorção tecnológica, têm, assim como (2) se referiu aos estabelecimentos penitenciários, um "sistema peculiar de vida interna (,..)" que "(...) configuram as condições propícias para a formação e manutenção de padrões culturais específicos (.,.)". Da mesma forma(1' afirmam que "grupos com "background" ocupacional semelhante tendem a desenvolver culturas próprias no interior das organizações".

No presente estudo, busquei compreender os universos simbólicos compartilhados pelos seus atores e os mecanismos de reforço dessa cultura. Esse resgate foi pautado nas próprias experiências dos enfermeiros intensivistas, nas expressões e compreensões de sua prática, onde o contexto em que se dá, foi parte integrante do estudo,

No desenvolver da pesquisa o aspecto da cultura do CTI que se mostrou mais significativo foi o processo de socialização dos novos membros, O CTI passava por um processo de demissão e admissão de um grande número de enfermeiros e esse se tornou, assim, o foco etnográfico do estudo.

Compreendendo o estudo etnográfico como um processo investigativo caracterizado por uma grande flexibilidade metodológica, os objetivos foram direcionados no próprio desenvolvimento da pesquisa, ficando assim constituídos:

- Descrever a subcultura dos enfermeiros intensivistas para melhor compreender sua prática,

- $\quad$ Compreender o processo de socialização dos novos membros na subcultura do Centro de Tratamento Intensivo.

\section{O CENTRO DE TRATAMENTO INTENSIVO}

Os trabalhos com temáticas relacionadas à Terapia Intensiva são, em grande parte, estudos quantitativos e que analisam procedimentos diagnóstico - terapêuticos de pacientes internados naquele setor.

$\mathrm{Na}$ análise retrospectiva (1975-1984) das pesquisas de enfermagem em Terapia Intensiva), concluem também que são, na maioria, pesquisas que adotam a abordagem quantitativa e que investigam, predominantemente, os procedimentos de enfermagem ou terapêuticos e de parâmetros fisiológicos de pacientes criticos.

No período de 1993-1996 foram encontrados na literatura 299 trabalhos de enfermagem com temáticas relativas à terapia intensiva. O levantamento bibliográfico realizado confirma as conclusões(3), ou seja, a produção científica sobre o tema é, na grande maioria, relacionada aos procedimentos diagnóstico-terapêuticos,

Na produção científica relativa a área de Terapia Intensiva podemos apontar aquelas que tiveram como foco a comunicação ${ }^{(4)}$, riscos e iatrogenias decorrentes do uso da tecnologia(8); a questão da humanização da assistência (6); a questão do estresse, aspectos emocionais e psiquiátricos, dos profissionais ou dos pacientes ${ }^{(7)}$; aspectos éticos ${ }^{(8-9)}$; a prática dos profissionais inten-sivistas $(10)$, a questão da avaliação do uso de tecnologia, de modelos administrativos e de assistência(11-31) São encontrados, também, trabalhos que têm como objetivo analisar a produção científica referente a CTI e outros que objetivavam a reflexão sobre questões teórico-meto-dológicas na realização de pesquisas neste setor ${ }^{(14-61)}$

São encontradas algumas pesquisas que adotam abordagens qualitativas para o estudo de questões fundamentais para a compreensão do cotidiano da terapia intensiva, numa perspectiva fenomenológica. $\left({ }^{16-17}\right.$; e outras abordagens qualitativas, como (18-19)

Alguns autores tratam o CTI na sua dimensão cultural, numa perspectiva etnográfica, identificando, significados, símbolos, valores, mitos e ritos compartilhados por pacientes ou profissionais(20-21)

No entanto, não foram encontrados estudos que tiveram como objetivo, descrever o. processo de socialização dos novos membros enfermeiros na cultura da terapia intensiva,

\section{A OPÇÃO METODOLÓGICA}

Esse estudo foi realizado numa tentativa de aproximação à perspectiva derivada da Antropologia Interpretativa, também denominada Antropologia pósmoderna, pautada no paradigma hermenêutico.

A Antropologia Interpretativa, pautada no paradigma hermenêutico, reconsidera verdades que a Antropologia acreditava eternas (22-23)

As questões demandadas pela hermenêutica estão pautadas-, entre outros princípios, na importância do contexto na compreensão do texto e na aceitação da intersubjetividade.

O Centro de Tratamento Intensivo, neste estudo, é compreendido como uma cultura, uma forma de expressão e manifestação de interações humanas, um universo de símbolos e significados peculiares entre seus membros, 
universo de símbolos e significados peculiares entre seus membros. Alguns desses significados são expressos através da linguagem e muitos são "taken for granted" e expressos, indiretamente, através das ações (24)

Esse trabalho, assim como os estudos organizacionais que tratam a cultura como um sistema de símbolos e significados, buscou descrever os sistemas temáticos de significados subjacentes às atividades dos intensivistas, com o objetivo de decifrá-los e compreendê-los.

Reportando-me à natureza do objeto estudado, ao objetivo desta pesquisa e aos pressupostos teóricometodologicos adotados considerei pertinente a opção por uma abordagem qualitativa tendo em vista que: o contexto da cena cultural foi considerado parte da pesquisa (25-26); o cotidiano do CTI foi descrito sob o ponto de vista dos enfermeiros intensivistas (27-29) não foram delimitadas hipóteses a priori(30)

Assim, optei por realizar um estudo etnográfico através de trabalho de campo, uma vez que é adequado para. compreender a complexidade dos fenômenos culturais da organização, sob a perspectiva de seus próprios membros, nos seus "próprios termos" (31)

Na ótica de (24), a etnografia é o trabalho de descrição de uma cultura a partir da visão de mundo do nativo dessa cultura. A etnografia busca a compreensão do significado de ações e eventos para os participantes, significados esses expressos através da linguagem ou indiretamente através de ações, constituindo-se num sistema de significados complexos. Esse sistema de significados constitui a cultura.

O estudo foi realizado no ano de 1997, em um CTI de um hospital público, universitário de grande porte, em Belo Horizonte.

Os dados foram coletados através de observação participante do cenário cultural $\mathrm{n}$ período da manhã, tarde e noite; entrevistas etnográficas realizadas junto aos enfermeiros do setor -os informantes do estudo - e análise de documentos tais como, normas, rotinas do setor e registro em prontuários com o objetivo de complementar as informações obtidas pelas outras técnicas e contextualizar e identificar aspectos históricos do setor. As entrevistas foram agendadas com os informantes e realizadas no CTI, em local privativo, garantindo o anonimato.

Foram realizadas análises de domínio, taxonômica, componencial e temática, implicando numa volta contínua às anotações de campo e registro das entrevistas etnográficas. Foi, também, utilizada a abordagem de inventário, no sentido de subsidiar a construção de um modelo das interações simbólicas da subcultura estudada.

A análise etnográfica é a busca da estrutura interna de uma cultura, suas partes e as relações entre essas partes, sob o ponto de vista dos nativos dessa cultura, denominados de informantes. Seu objetivo principal é a descoberta de "significados culturais".

A análise de dados etnográficos é um processo contínuo, cíclico e simultâneo á coleta de dados através da qual são identificados os significados culturais (24)

O objetivo da análise de domínio é identificar categorias de significados culturais. Os domínios constituídos de três elementos básicos: termos inclusos (denominação das categorias menores); termos encobertos (nome dos domínios) e relação semântica entre os termos (24)

A organização interna do domínio constitui a análise taxonômica dos dados etnográficos. A taxonomia estabelece a relação entre os termos inclusos de um domínio cultural.

A dimensão de contraste entre os símbolos de uma cultura emerge das perguntas de contraste. A busca dessas diferenças de significados entre termos populares, também chamadas de componentes de significado ou dimensões de contraste, dá origem à análise componencial.

Uma visão mais ampla da cultura é, segundo o autor, obtida pela identificação de temas recorrentes, através do processo de imersão nos dados e validação com os informantes. Os temas culturais são definidos por SPRADLEY (1980:141) como "principios cognitivos recorrentes num determinado número de domínios, tácitos ou explícitos, mostrando o relacionamento entre os subsistemas de significado cultural".

$\mathrm{Na}$ abordagem de inventário o etnógrafo busca agrupar os dominios identificados em categorias relativas aos vários eventos culturais tais como, parentesco, relações sociais, artefatos, entre outros. O etnógrafo busca descobrir os sistemas conceituais (temas culturais) que os membros da cultura utilizam para conectar esses domínios, obtendo uma visão holística da cultura.

No desenvolver da etnografia, a grande variação e complexidade de situações encontradas no campo de pesquisa colocaram-me frente a algumas questões éticas.

No enfrentamento dessas questões, adotei alguns princípios éticos preconizados por (24) pautados nos Princípios de Responsabilidade Erofissional da Associação Antropológica Americana (1971), tais como, o direito do informante de conhecer os objetivos e resultados do estudo, o respeito aos seus interesses e à sua privacidade, o anonimato, a participação voluntária dos informantes e a utilização científica dos resultados.

No presente estudo, o trabalho de campo foi desenvolvido de forma revelada, ou seja, com conhecimento por parte dos participantes, do objetivo geral da pesquisa e da identidade da pesquisadora. $\mathrm{Da}$ mesma forma, o relatório final foi divulgado entre os participantes da pesquisa.

\section{A SOCIALIZAÇÃO DOS NOVOS MEMBROS}

A socialização dos novos membros é caracterizada pelos informantes como um processo doloroso, dificil e muitas vezes solitário. Segundo os informantes é o processo pelo qual são repassados os conhecimentos não só técnicos, como a "tradição" da terapia intensiva.

\section{As formas:}

Segundo os informantes a socialização dos novos membros se dá em duas etapas, ou seja, antes de entrar para o setor e dentro do CTI. Na Taxonomia $\mathrm{n}^{\circ} 1$ estão descritas as formas de socialização dos novos membros daquela subcultura. 
TAXONOMIA № 1: FORMAS DE SOCIALIZAÇÃO DOS NOVOS MEMBROS DA ENFERMAGEM

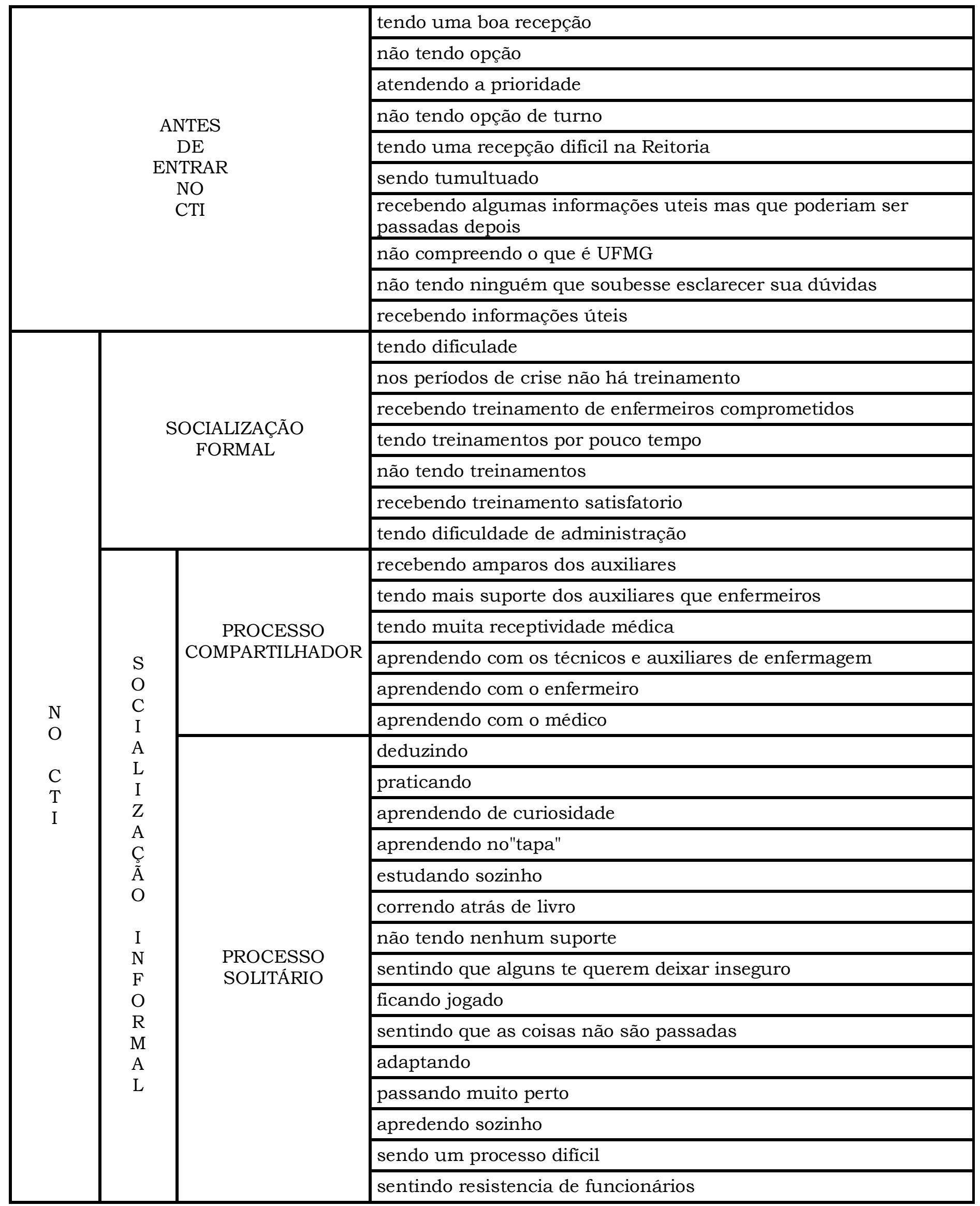


Antes de entrar para o CTI, o novo funcionário é recebido de forma tumultuada, recebendo algumas informações úteis, mas outras inúteis. Não entende o hospital dentro do contexto da universidade, ficando com muitas dúvidas não esclarecidas.

Ao entrar para o CTI, o novo membro passa por um processo de socialização formal, através de treinamentos, com o conteúdo, eminentemente técnico. Por outro lado, o conhecimento cultural é, também repassado, através de um processo informal de socialização. A socialização informal se dá de forma compartilhada, onde há uma participação efetiva dos"antigos", como também, através de um processo solitário, onde o novo membro "vai aprendendo que nem a vida", "deduzindo", "correndo atrás", "adaptando".

\section{Os sentimentos e as causas}

O processo de socialização dos novos membros é permeado de sentimentos de incompetência, insegurança, angústia, preocupação e muito medo, Medo de "não dar conta", de errar, "da experiência dos outros", do desconhecido, entre outros (Taxonomia $\mathrm{n}^{\circ}$ 2)

TAXONOMIA N² 2: TIPOS DE SENTIMENTOS DOS ENFERMEIROS QUANDO ENTRAM PARA O CTI

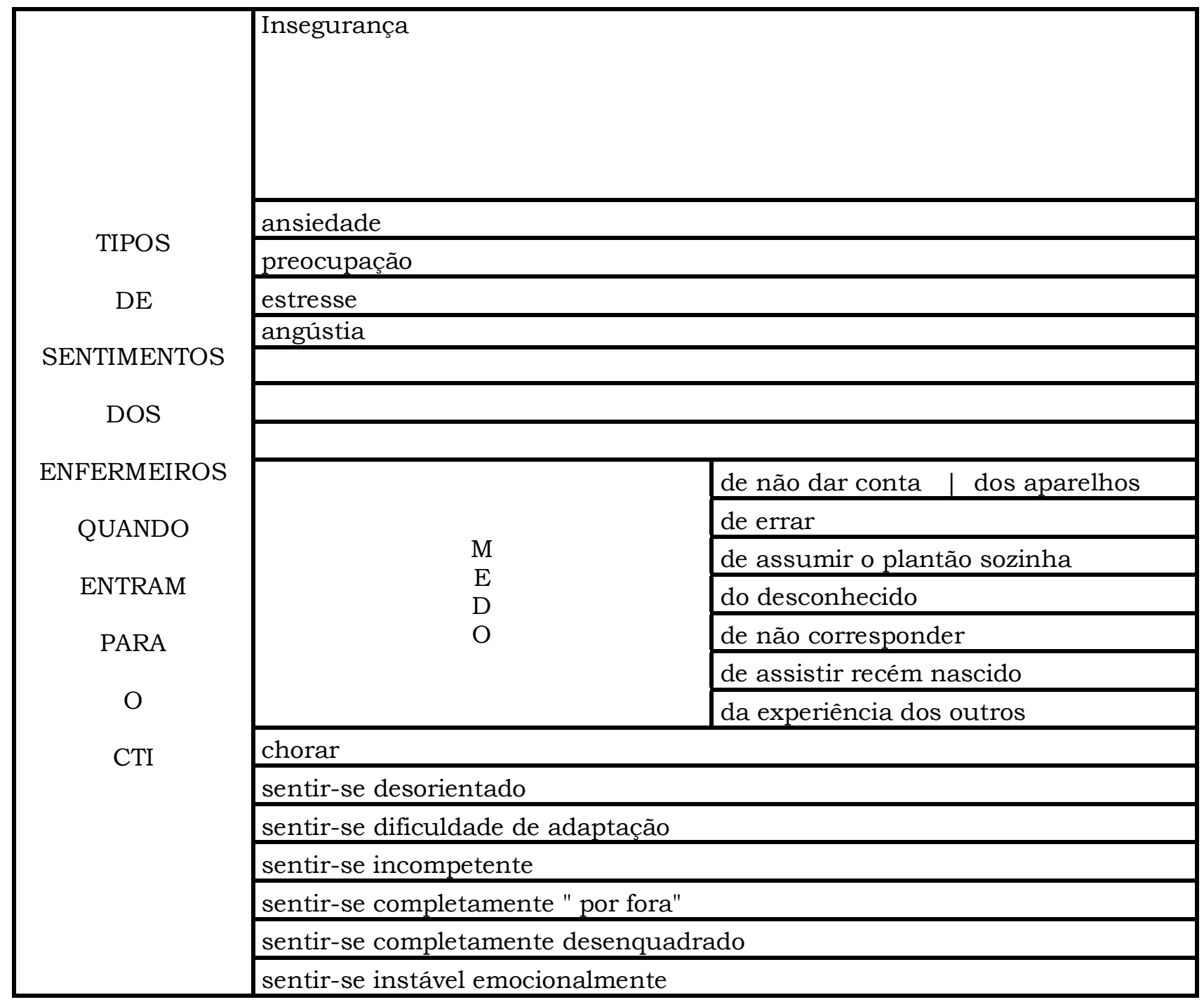

No relato abaixo estão evidenciados os tipos de sentimentos dos enfermeiros quando entram para o CTI:

"Quando vim para o CTI eu fui obrigada, porque a necessidade do setor previa um enfermeiro a noite. Eu vim insegura. Medo de assumir o plantão sozinha, assumir as atividades específicas do enfermeiro sozinha". 


\begin{tabular}{|c|c|c|c|c|c|c|c|}
\hline \multicolumn{8}{|c|}{ CAUSAS DOS SENTIMENTO DOS ENFERMEIROS QUANDO ENTRAM PARA O CTI } \\
\hline \multirow{2}{*}{$\begin{array}{c}\text { RELATIVAS AO } \\
\text { PROCESSO DE } \\
\text { FORMAČÃO } \\
\text { PROFISSIONAL } \\
\end{array}$} & \multirow{2}{*}{$\begin{array}{c}\text { RELATIVAS AO } \\
\text { ATRIBUTOS DA } \\
\text { ASSISTENCIA DE CTI }\end{array}$} & \multirow{2}{*}{$\begin{array}{c}\text { RELATTVAS AO } \\
\text { PROCESSO DE } \\
\text { ADMISSÃO NO } \\
\text { HOSPITAL }\end{array}$} & \multirow{2}{*}{$\begin{array}{c}\text { RELATIVAS AO } \\
\text { ATRIBUTOS DOS } \\
\text { PACIENTES DE CTI }\end{array}$} & \multicolumn{4}{|c|}{ RELATIVAS AO ASPECTOS ADMINISTRATIVOS } \\
\hline & & & & RECURSOS HUMANOS & \multicolumn{3}{|c|}{$\begin{array}{l}\text { RECURSOS FÍSICOS E } \\
\text { MATERIAIS }\end{array}$} \\
\hline não saber nada de CTI & exigir muita responsabilidade & não ter treinamento & $\begin{array}{l}\text { serem os pacientes muito } \\
\text { complexos }\end{array}$ & $\begin{array}{l}\text { ter que ficar atento, ao } \\
\text { relacionamento }\end{array}$ & \multicolumn{3}{|c|}{ ter muitas coisas novas } \\
\hline $\begin{array}{l}\text { ter visitado o CTI apenas uma } \\
\text { vez }\end{array}$ & $\begin{array}{l}\text { ter que atender tudo a } \\
\text { qualquer hora }\end{array}$ & ir para o CTI obrigado & & $\begin{array}{l}\text { ter que ficar atento aos } \\
\text { funcionários }\end{array}$ & \multicolumn{3}{|c|}{ ter que ficar atento às aparelhagens } \\
\hline não ter CTI na Escola & ser muito complexa & & não ter limite de idade & $\begin{array}{l}\text { ter que ficar atento à } \\
\text { realizaçāo das técnicas }\end{array}$ & \multirow[t]{8}{*}{$\begin{array}{l}\text { detectar } \\
\text { falhas }\end{array}$} & \multirow[t]{8}{*}{\begin{tabular}{|l}
$\begin{array}{l}\text { não } \\
\text { danificá- } \\
\text { los }\end{array}$ \\
\end{tabular}} & \multirow{8}{*}{$\begin{array}{l}\text { observar } \\
\text { riscos } \\
\text { para } \\
\text { ora } \\
\text { paciente }\end{array}$} \\
\hline não ter estudado CTI & exigir muito da gente & ficar abandonado no CTI & ser o paciente muito invadido & & & & \\
\hline $\begin{array}{l}\text { não saber agir em situaçăo de } \\
\text { urgencia }\end{array}$ & $\begin{array}{l}\text { serem os procedimentos muito } \\
\text { invasivos }\end{array}$ & $\begin{array}{l}\text { ter treinamento por pouco } \\
\text { tempo }\end{array}$ & ser maior o risco de vida & & & & \\
\hline $\begin{array}{l}\text { não ter nenhuma base teórica } \\
\text { de CTI }\end{array}$ & ter cautela dobrada & ter que assumir o CTI rápido & & & & & \\
\hline ser o CTI uma realidade nova & & ter que ser por si mesmo & & & & & \\
\hline \multicolumn{5}{|l|}{$\begin{array}{l}\text { ser o CTI uma lacuna no } \\
\text { processo de formação do } \\
\text { enfermeiro }\end{array}$} & & & \\
\hline \multicolumn{5}{|l|}{$\begin{array}{l}\text { não ter estágio de CTI na } \\
\text { Escola }\end{array}$} & & & \\
\hline não dominar as técnicas de CTI & & & & & & & \\
\hline
\end{tabular}


As causas dos sentimentos dos enfermeiros quando entram para o CTI estão relacionadas na Taxonomia $\mathrm{n}^{\circ} 3$.

Como pode ser observado nos relatos, as causas desses sentimentos relacionam-se ao processo de formação do enfermeiro, às peculiaridades da assistência de terapia intensiva, ao processo de admissão no hospital, ao perfil do paciente usualmente admitido em CTI, bem como aos aspectos administrativos do setor.

"Não tinha base teórica nenhuma e prática nenhuma também. É uma lacuna que todo mundo reclama, inclusive o pessoal da federal, reclama muito isso".
"A gente não tem, infelizmente, formação acadêmica, nem de auxiliar, nem de atendente, nem de enfermeiro, a gente não tem CTI. Até o próprio médico. Tem que ser por si mesmo, procurar estágio".

\section{Os estágios}

O processo de socialização dos novos membros, na perspectiva dos intensivistas é constituído de etapas, como pode ser observado na Taxonomia $n^{\circ} 4$

\section{TAXONOMIA N² 4: ESTÁGIOS DO PROCESSO DE SOCIALIZAÇÃO DOS NOVOS MEMBROS}

\begin{tabular}{|c|c|}
\hline \multirow{10}{*}{$\begin{array}{l}\mathrm{O} \\
\mathrm{N} \\
\mathrm{I} \\
\mathrm{P} \\
\mathrm{O} \\
\mathrm{T} \\
\hat{\mathrm{E}} \\
\mathrm{N} \\
\mathrm{C} \\
\mathrm{I} \\
\mathrm{A}\end{array}$} & sentimento de poder curar tudo \\
\hline & sentimento de poder salvar tudo \\
\hline & mal emprego da tecnologia \\
\hline & uso da tecnologia de forma indiscriminada \\
\hline & não seleção de quem vai receber investimento \\
\hline & admissão de pacientes sem indicação \\
\hline & desempenho do papel de ressuscitador \\
\hline & ter máscara de frio \\
\hline & achar que o CTI é o templo do saber \\
\hline & gastar muito material \\
\hline \multirow{7}{*}{$\begin{array}{l}\mathrm{J} \\
\mathrm{U} \\
\mathrm{I} \\
\mathrm{Z}\end{array}$} & querer aprender com os antigos \\
\hline & maior critério no uso de tecnologia \\
\hline & onipotência desafiada \\
\hline & compreensão de não poder salvar todo mundo \\
\hline & compreensão de não poder mudar o curso de certas doenças \\
\hline & crítica às deficiências do serviço \\
\hline & ser humilde \\
\hline
\end{tabular}

Ao ser socializado, o novo membro do CTI passa por dois estágios: o da onipotência e o do juiz. O primeiro é caracterizado como uma fase na qual o novo membro tem o sentimento de poder curar todas as doenças, usa os recursos tecnológicos de forma indiscriminada, adotando uma máscara de frio com o significado de "estar tudo sob controle".

No segundo estágio, passa a definir critérios para a utilização da tecnologia disponível, sente que sua onipotência é freqüentemente desafiada, compreendendo que não tem o poder de cura e de mudar o curso de certas doenças. A partir deste estágio, adota uma postura mais humilde e de crítica às deficiências do setor:
"Eu acho que tem duas fases em terapia intensiva. Por exemplo, seria a onipo-tência, a sensação de que podem curar tudo, salvar tudo. O CTI propicia um ambiente adequado para isso, um ambiente onde você dispõe de tecnologia, onde você faz o papel de ressuscitados. Você é capaz de ressuscitar as pessoas, tem essa parte de sentir onipotente, você é capaz de tratar ele, resgatar tudo. Depois eu diria que essa onipotência começa a ser desafiada, quando você percebe que apesar da utilização de todos os recursos, você acaba não conseguindo salvar todo mundo, não 
modificar o curso de muitas doenças. Então entra uma fase onde você passa a criterizar melhor a aplicação de recursos, seria a fase do juiz".

\section{Os fatores facilitadores}

A socialização é caracterizada pelos informantes como um processo doloroso, permeado de sentimentos de angústia, insegurança, medo. No entanto, existem situações que, segundo os membros daquela subcultura, facilitam a entrada no novo membro no grupo. Essas situações estão relacionadas à vivência anterior do cotidiano da terapia intensiva, ao contato anterior com funcionários do setor e, principalmente à possibilidade da introdução de conteúdos programáticos relativos a CTI na graduação dos enfermeiros, como pode ser observado no relato:

"Você tem que ver esse lado. Tem que inserir isso enquanto você estiver fazendo o curso de graduação para despertar aquilo que no futuro vai te engajar mesmo na carreira de intensivista. CTI não é aquilo que a gente imagina".

\section{CONCLUSÃO}

Ao concluir esse estudo, foi identificado que, no decorrer da trajetória histórica do CTI, foi emergindo uma identidade organizacional da terapia intensiva, que certamente sofreu influências do modelo de assistência que lhe deu origem - o "Cuidado Progressivo ao Paciente" -e das características pessoais de seus fundadores onde é evidente o valor da organização, do sentido de equipe, do estabelecimento de normas rígidas de condutas técnicas e administrativas.

Nessa trajetória ficou evidente que, na atualidade, o setor foi descaracterizado enquanto um CTI, uma vez que é cada vez mais dificil repassar seus valores, mitos e ritos em decorrência de um processo significativo de rotatividade de seus membros. As razões da alta rotatividade estão relacionadas às condições salariais, às peculiaridades do trabalho em CTI - "cansativo" e "estressante" -, às peculiaridades do setor "fechado" e "isolado".

A crise administrativa pela qual vem passando o hospital, em geral, e o CTI, em particular, é caracterizada pela "perda das referências profissionais" - heróis culturais - que valorizavam o "compromisso" com o serviço, o "vestir a camisa", a "organização", a "disciplina", a "qualidade" e o sentido de unidade, de equipe.

Os antigos, que são a minoria, tentam, algumas vezes, em vão, reproduzir os "velhos" valores de qualidade, da organização, do compromisso, do sentido da unidade para os novos membros que permanecem ali por tão pouco tempo. A necessidade de organização é considerada fundamental para que o CTI possa ser funcional frente à sua dinâmica ágil. Tal característica, às vezes, é determinante do sucesso no atendimento aos pacientes.

O processo de socialização é o processo pelo qual o novo membro incorpora os universos simbólicos da subcultura do CTI. Neste processo são reproduzidos os valores de qualidade, da disciplina, do compromisso ("do vestir a camisa"), do sentido da unidade que é muito mais do que o significado de trabalho de equipe, o valor das normas de condutas rígidas e de organização. Esses valores são considerados condições indispensáveis para que um setor possa ser considerado um CTI.

O processo de incorporação dos universos simbólicos é permeado de sentimentos de insegurança, ansiedade, angústia e de incompetência e medo. Medo "de não dar conta", "do desconhecido", "da experiência dos outros", "de errar". E errar muitas vezes...

As causas destes sentimentos, independentemente de sua natureza, têm um forte componente -a não vivência anterior, uma lacuna na formação profissional. Outras causas estão relacionadas às peculiaridades da assistência, do paciente e do trabalho em terapia intensiva, onde estão presentes os significados de complexidade, estresse, instabilidade, risco e isolamento.

Entrar para o CTI é, às vezes mais opção institucional que pessoal. Frente às adversidades do ambiente interno, alguns não se adaptam, visto que é um setor fechado, isolado onde o trabalho é cansativo e estressante. Por outro lado, requer do novo membro características pessoais e profissionais, tais como compromisso, dinamismo, organização, responsabilidade, raciocínio rápido.

No processo de tornar-se um intensivista o novo membro passa pelo estágio da onipotência, quando acredita que pode "curar. tudo", "salvar tudo",

"desempenhado o papel de ressuscitador", "empregando mal a tecnologia", e não "selecionando quem vai receber o investimento". Adota a "máscara de frio" e encara o CTI como o "templo do saber".

Com o passar do tempo, sente sua onipotência desafiada e adota a humildade frente à compreensão do limite humano de intervir no curso natural de certas doenças.

O processo de interiorização dos universos simbólicos organizacionais se dá formal e informalmente mas é sempre referido como árduo, penoso; muitas vezes solitário; algumas vezes compartilhado.

Quando solitário, requer o desafio do correr atrás, de buscar caminhos, aprender no "tapa", perguntando ali, observando acolá. Quando compartilhado é solidariedade, compreensão, é o dividir do saber, do prestígio e do "status", é o compartilhar do saber técnico e cultural. Assim, o enfermeiro, também, aprende com outro enfermeiro e o auxiliar, tendo, às vezes, mais suporte do segundo que do primeiro.

O sofrido "tornar-se um intensivista" pode ser facilitado através de algumas condições, basicamente relacionadas à experiências anteriores no setor e de atender pacientes graves. A introdução de conteúdos 
teórico-práticos relativos à assistência ao paciente crítico, internados em CTI, na graduação dos profissionais de saúde é o fator facilitador mais significativo para o grupo estudado.

A cultura, enquanto um sistema compartilhado de significados, é definida, revisada e aprendida pelo novos membros nas relações sociais do grupo.

Fazendo inferências culturais o novo membro aprende aquele conhecimento comum, repassado através da linguagem, de atitudes do grupo (comportamento cultural), na observação e participação de ritos (treinamentos, a hora do cafezinho), no contexto das interações sociais institucionais (nos corredores, na copa, no posto de enfermagem nas reuniões clínicas e nas passagens de plantão).

Nesse processo de interiorização de símbolos e significados, é aprendido, às vezes a dura penas, sobreviver no isolamento, transferir emoções e uma nova dimensão do atendimento, onde a prioridade é a vida, é manter a estabilidade, é investir no imponderável.

\section{REFERÊNCIAS BIBLIOGRÁFICAS}

(1) Fleury MTL, Fischer RM, coordenador. Cultura e poder nas organizações. São Paulo: Atlas; 1989.

(2) Fischer RM. O circulo do poder: as práticas invisíveis de sujeição nas organizações complexas. In: Fleury MTL, Fischer RM, coordenador. Cultura e poder nas organizações. São Paulo: Atlas; 1989. p. 65-88,

(3) Koizumi MS, Miyadahira AMK, Takahashi EIU Análise retrospectiva das pesquisas de enfermagem em terapia intensiva: 1975.1984. Rev Esc Enferm USP 1986; 20(1): 5-17.

(4) Leathart AJ. Communication and socialisation: an exploratory study and explanation for nurse-pacient communication in an ITU. Intensive Crit Care Nurs 1994; 10(2): 93-104.

(5) Padilha KG. Des-cuidar: as representações sociais dos enfermeiros de UTI sobre as ocorrências iatrogenicas de enfermagem. (Tese] São Paulo (SP) - Escola de Enfermagem da Universidade de São Paulo; 1994.

(6) Ortiz-Pruitt J. Physical restraint of critically ill patients: a human issue. Crit Care Nurs Clin North Am 1995; 7(2): 36373 .

(7) Cutler L, Garner M. Reducing relocation stress after discharge from the intensive therapy unit. Intensive Crit Care Nurs 1995; 11(6): 333-5.

(8) Drought TS, Liaschenko J. Ethical practice in a technological age. Crit Care Nurs Clin North Am 1995; 7(2): 297-304.

(9) Rushton $\mathrm{CH}$. Creating an ethical pratice environment: a focus on advocacy. Crit Care Nurs Clin North Am 1995; 7(2): 387-97.

(10) Ehrenfeld M, Bar-Tal Y. Identifying the coping behaviours used

by nurses in intensive care. Nurs Stand 1995; 9(32): 27-30,

(11) Shortell SM, Zimmerman JL, Rosseau DM, Gillies RR, Wagner DP, Prapei EA, Kraus WA. The performance of intensive care units: does good management make a difference? Med Care 1994; 32(5): 508-25.

(12) Gordon M, Hiltunen E. Higt frequency: treatment priority nursing diagnoses in critical care. Nurs Diagn 1995; 6(4): 143-54.
(13) Locsin RC, Machine technologies and caring in nursing. Image $J$ Nurs Sch 1995; 27(3): 201-3,

(14) Riegel B, Banasek JL, Barnsteiner J, Beecroft P, Kern L, Lindguist R, Prevost S, et al. Reviews and summaries of research related to AACN 1980 research priorities: contextual topics, Am J Crit Care 1994; 3(4): 260-6.

(15) Suominem T, Leino-Kilpi H. There is very little European research on intensive care nursing, Intensive Crit Care Nurs 1995; 11(5): 244-51,

(16) Walters AJ. An interpretative study of the clinical pratice of critical care nurses. Contemp Nurse 1994(a); 3(1):21-5.

(17) Walters AJ. The comforting role in critical care nursing practice: a fhenomenological interpretation. Int $\mathrm{J}$ Nurs Stud 1994(b); 31(6): 607-16

(18) Jezewski MA. Do-not resuscitate status: conflict and culture brokering in critical care units. Heart Lung 1994; 23(6): 458-65.

(19) Spencer L. How do nurses deal with own grief when dies on an intensive care unit, and what help can be given to enable them to overcome their grief effectively? J Adv Nurs 1994; 19(6): 1141-50

(20) Henderson JN. The culture of special care unit: an anthropological perspective on ethnographic research in nursing home settings. Alzheimer Dis Assoc Disord 1994; 8 (Suppl. 1): 410-6.

(21) Chase SK. The social context of critical care clinical judgment. Heart Lung 1995; 24(2): 154-62.

(22) Oliveira RC, Sobre o pensamento antropológico, Rio de Janeiro: Tempo Brasileiro, 1988.

(23) Caldeira TPR. A presença do autor e a pós-modernidade em antropologia. Novos Est CEBRAP 1988; (21):133-57.

(24) Spradley J. The ethnographic interview. New York: Holt Rinehart \& Winston; 1979.

(25) Field PA, Morse JM. Nursing research: the application of qualitative approaches. London: Croom Helm; 1985.

(26) Trivinos ANS. Introdução á pesquisa em ciências sociais: a pesquisa qualitativa em educação. São Paulo: Atlas; 1987.

(27) Ludke M, Andre MEVA. Pesquisa em educação: abordagem qualitativas. São Paulo: EPU; 1986,

(28) Hammersley M, Atkinson P Ethnography: principles in practice. New York: Tavistock; 1983.

(29) Geertz C. A interpretação das culturas. Rio de Janeiro: Guanabara; 1989.

(30) Evertson CM, Green JL. La observation como indagación y método In: Wittrock MC. La investigación de Ia ensenanza, [II, Métodos cualitativos y de observación]. Madrid: Centro de Publicación del Ministerio de Educación y Ciência; 1989, cap.5. p. 303-421.

(31) Gregory KL. Native-view paradigms; multiple cultures and culture conflicts in organizations. Adm Sci Q 1983; 28:359-76.

Artigo recebido em $28 / 03 / 00$

Artigo aprovado em 21108101 